\title{
Comparison of Risk Factors for Trastuzumab-Related Cardiotoxicity in Elderly and Non-Elderly Breast Cancer Patients
}

\author{
Nobuyoshi Kittaka ${ }^{1 *}$, Mikio Mukai ${ }^{2}$, Reiko Fukui ${ }^{1}$, Yoko Hashimoto ${ }^{1}$, Makoto Ishitobi ${ }^{1}$, Takahiro Nakayama ${ }^{1}$ and Yasuhiro Tamaki $^{1}$ \\ ${ }^{1}$ Department of Breast and Endocrine Surgery, Osaka International Cancer Institute, Chuo-ku, Japan \\ ${ }^{2}$ Department of Onco-Cardiology, Osaka International Cancer Institute, Chuo-ku, Japan
}

*Corresponding author: Nobuyoshi Kittaka, Department of Breast and Endocrine Surgery, Osaka International Cancer Institute, 3-1-69 Otemae, Chuo-ku, Osaka 541-8567, Japan, Tel: +81-06-6972-1181; Fax: +81-06-6981-8055; E-mail: kittaka-no@mc.pref.osaka.jp

Received: 23 Jan, 2018 | Accepted: 30 Jan, 2018 | Published: 31 Jan, 2018

Citation: Kittaka N, Mukai M, Fukui R, Hashimoto $\mathrm{Y}$, Ishitobi $M$, et al. (2018) Comparison of Risk Factors for Trastuzumab-Related Cardiotoxicity in Elderly and Non-Elderly Breast Cancer Patients. Clin Res Open Access 4(1): dx.doi.org/10.16966/2469-6714.131

Copyright: () 2018 Kittaka N, et al. This is an open-access article distributed under the terms of the Creative Commons Attribution License, which permits unrestricted use, distribution, and reproduction in any medium, provided the original author and source are credited.

\begin{abstract}
Background and Objective: Although trastuzumab-based treatment benefits patients with HER2-positive early and metastatic breast cancer improving both disease free and overall survival, cardiotoxicity has been a crucial issue. The purpose of this study is to investigate predictive risk factors for trastuzumab-related cardiotoxicity in elderly and non-elderly patients.
\end{abstract}

Patients and Methods: We reviewed medical records of 260 patients treated with trastuzumab for breast cancer from January 2008 to March 2015. Cardiotoxicity was defined as a decrease of the left ventricular ejection fraction (LVEF) either $>10 \%$ from the baseline or to a value $<53 \%$. The independent risk factors were determined from clinicopathological and echocardiographic parameters, using univariate and multivariate logistic models, and Receiver Operating Characteristic (ROC) curve analysis.

Results: Fifty-seven elderly ( $\geqq 65$ years old) and 203 non-elderly patients ( $\leqq 64$ years old) were included. The baseline LVEF was significantly related to cardiotoxicity both in elderly and non-elderly patients. The optimal cutoff values were $67 \%$ for elderly (the area under the curve at 0.77 ) and $66 \%$ for non-elderly patients (the area under the curve at 0.76 ). The baseline LVEF value of less than $67 \%$ and $66 \%$ in elderly patients (or $5.59,95 \% \mathrm{Cl}: 1.08-33.24 ; p=0.04$ ) and non-elderly patients (OR $4.61,95 \% \mathrm{Cl}: 1.48-17.50 ; p<0.01$ ) respectively was a significant predictive factor of trastuzumab-related cardiotoxicity.

Conclusions: A lower value of baseline LVEF may be the common risk factor for trastuzumab-related cardiotoxicity both in elderly and nonelderly breast cancer patients.

Keywords: Trastuzumab; Cardiotoxicity; Risk factor; Elderly patients

\section{Introduction}

Epidermal growth factor receptor 2 (HER2) is expressed in approximately $25 \%$ of all breast cancers [1]. Trastuzumabbased chemotherapy significantly improves the prognostic outcomes of patients with early stage and metastatic HER2positive breast cancer $[2,3]$. The use of trastuzumab is related to an increased risk of cardiotoxicity, which is attributed to blocking HER2 signaling in cardiac myocytes [4]. Cardiotoxity ranges from $2 \%$ to $7 \%$ for trastuzumab monotherapy, $2 \%$ to $13 \%$ for trastuzumab combined with taxane, and can be as high as $27 \%$ in combination with anthracycline [5].

Previous reports of large-scaled clinical trials have suggested that older age and cardiac comorbidity are important risk factors associated with cardiotoxity among trastuzumab-treated patients [6,7]. Therefore, trastuzumab-based chemotherapy is preferentially avoided for elderly patients in consideration of the risk of cardiotoxicity, while some can safely receive the treatment with trastuzumab. However, little is known regarding what factors are associated with cardiotoxicity in elderly patients. A few studies have suggested that some clinical factors such as history of cardiac disease, diabetes, hypertension, and obesity are associated with trastuzumab-related cardiotoxicity $[8,9]$, but the potential risk factor in elderly patients warrant further investigations.

For decades, echocardiography has been a major modality for evaluation of cardiac function. Left ventricular ejection fraction (LVEF) is a crucial parameter recommended for use in clinical decision-making, diagnosis and outcome evaluation by major guidelines and trials $[10,11]$. Previously reported, trastuzumab-related cardiotoxicity occurs more frequently in patients with a lower baseline LVEF [12,13]. It is not yet clear whether the lower baseline LVEF is an independent risk factor in elderly people.

In this study, we reviewed medical records of breast cancer patients treated with trastuzumab retrospectively, and examined clinical and cardio functional parameters to find 
potential risk factors of trastuzumab-related cardiotoxicity in elderly patients by comparing with those in non-elderly ones.

\section{Patients and Methods}

\section{Patient population and methods of treatment and observation}

This was a single institutional retrospective study. Medical records of patients who were treated at the Department of Breast and Endocrine Surgery, Osaka Medical Center for Cancer and Cardiovascular Diseases from January 2008 to March 2015 were reviewed. HER2 positivity was defined as $3+$ staining in immunochemistry or a positive result of fluorescence in situ hybridization (FISH) in the case of $2+$ staining according to the ASCO/CAP guideline. Patients who were treated with trastuzumab for any indication (adjuvant and metastatic) were included in the study. Patients who had been given trastuzumab as an adjuvant treatment previously and were treated for recurrence during the period were also included. An elderly patient was defined as one who was 65 years old or more, according to the statement by the International Conference on Harmonization [14], on the first day of starting the treatment. Trastuzumab was administered every 3 weeks (at an $8 \mathrm{mg} / \mathrm{kg}$ loading dose followed by $6 \mathrm{mg} / \mathrm{kg}$ ) or weekly (at a $4 \mathrm{mg} / \mathrm{kg}$ loading dose followed by $2 \mathrm{mg} / \mathrm{kg}$ ). All patients in this study were assessed for LVEF by $2 \mathrm{D}$ echocardiography scan before and every three months after starting the treatment by a team of experienced cardiologists. Cardiotoxicity during the treatment was defined as a decrease of LVEF of $>10 \%$ or to a value $<53 \%$ according to the definition by the American Society of Echocardiography and the European Association of Cardiovascular Imaging [15]. We also examined the E/e' ratio (the ratio of early diastolic transmitral flow velocity to mitral annular velocity at the lateral wall), which is reported to be an echocardiographic parameter for predicting cardiotoxicity of trastuzumab treatment [16]. Other clinicopathological data including age at treatment initiation, body mass index (BMI), estrogen receptor (ER) status, history of hypertension, diabetes mellitus, hyperlipidemia, hyperuricemia and cardiovascularrelated disease, and previous therapeutic regimen or radiation therapy were retrieved from the medical records.

\section{Statistical analysis}

The information was recorded in an Excel database and analyzed using JMP pro statistical software (Version 11.0.0, SAS Institute Inc., and Cary, NC, USA). To assess baseline characteristics, categorical variables were compared using the Fisher's exact probability test while continuous variables were analyzed using the Mann-Whitney U-test whenever appropriate. Odds ratios (or) and their 95\% confidence intervals calculated from logistic regression analysis were used to evaluate the relationship of risk factors to the development of cardiotoxicity. All relevant variables being significant $(\mathrm{p}<0.05)$ or showing a tendency towards significance in the univariate analysis were analyzed using a multivariate logistic model.
Receiver Operating Characteristic (ROC) curve analysis was applied to determine the cutoff value of the baseline LVEF value for prediction of cardiotoxicity.

\section{Results}

A total of 260 patients including 57 elderly and 203 nonelderly ( $\leqq 64$ years old) patients were enrolled in this study. Table $1 \mathrm{~A}$ and $1 \mathrm{~B}$ show the baseline clinicopathological characteristics of those. Eight (14\%) of the elderly patients and 19 (9\%) of the non-elderly patients had trastuzumab-related cardiotoxicity. In elderly patients, the average LVEF value at baseline was significantly lower in those with cardiotoxicity than those without $(p=0.02)$. In non-elderly patients, the average LVEF values at baseline $(p<0.01), \mathrm{BMI}(p=0.04)$, and E/e' at baseline $(p=0.05)$, were significantly lower in those with cardiotoxicity than those without.

In the univariate analysis table 2, low LVEF values at baseline $(p=0.05)$, some cardiovascular diseases $(p=0.16)$, history of anthracycline exposure $(p=0.25)$, and adjuvant usage of trastsuzumab $(p=0.26)$ were relatively associated with cardiotoxicity in elderly patients. On the other hand, in nonelderly patients, low LVEF values at baseline $(p<0.01)$, low BMI $(p=0.02)$, and low E/e' at baseline $(p=0.03)$ were identified as

Table 1A: Comparison of the factors between non-cardiotoxicity group and cardiotoxicity group in elderly patients.

\begin{tabular}{|c|c|c|c|c|}
\hline Factors & & $\begin{array}{c}\text { Non- } \\
\text { cardiotoxicity } \\
\text { group/(n=49) }\end{array}$ & $\begin{array}{l}\text { Cardiotoxicity } \\
\text { group/(n=8) }\end{array}$ & $\begin{array}{c}p \\
\text { value }\end{array}$ \\
\hline Age (year)* & & $70.1 \pm 5.0$ & $69.6 \pm 3.5$ & $1.00^{b}$ \\
\hline BMI $\left(\mathrm{kg} / \mathrm{m}^{2}\right)^{\star}$ & & $21.5 \pm 3.8$ & $21.4 \pm 2.7$ & $0.89^{b}$ \\
\hline \multirow{2}{*}{ ER status } & Negative & 24 & 3 & \multirow{2}{*}{$0.71^{a}$} \\
\hline & Positive & 25 & 5 & \\
\hline \multirow{3}{*}{$\begin{array}{l}\text { Radiation } \\
\text { therapy }\end{array}$} & No & 22 & 5 & \multirow{2}{*}{$0.71^{a}$} \\
\hline & Left & 13 & 1 & \\
\hline & Right & 14 & 2 & \\
\hline \multirow{2}{*}{ Hypertension } & No & 32 & 5 & \multirow{2}{*}{$1.00^{\mathrm{a}}$} \\
\hline & Yes & 17 & 3 & \\
\hline \multirow{2}{*}{ Diabetes } & No & 43 & 7 & \multirow{2}{*}{$1.00^{\mathrm{a}}$} \\
\hline & Yes & 6 & 1 & \\
\hline \multirow{2}{*}{ Hyperlipidemia } & No & 31 & 5 & \multirow{2}{*}{$1.00^{\mathrm{a}}$} \\
\hline & Yes & 18 & 3 & \\
\hline \multirow{2}{*}{ Hyperuricemia } & No & 40 & 7 & \multirow{2}{*}{$1.00^{\mathrm{a}}$} \\
\hline & Yes & 9 & 1 & \\
\hline \multirow{2}{*}{$\begin{array}{l}\text { Cardiovascular } \\
\text { disease }\end{array}$} & No & 42 & 5 & \multirow{2}{*}{$0.15^{\mathrm{a}}$} \\
\hline & Yes & 7 & 3 & \\
\hline \multirow{2}{*}{$\begin{array}{l}\text { Anthracyclines } \\
\text { use }\end{array}$} & No & 29 & 3 & \multirow{2}{*}{$0.28^{a}$} \\
\hline & Yes & 20 & 5 & \\
\hline \multirow{2}{*}{ Taxanes use } & No & 20 & 4 & \multirow{2}{*}{$0.78^{a}$} \\
\hline & Yes & 29 & 4 & \\
\hline \multirow{2}{*}{$\begin{array}{l}\text { Trastsuzumab } \\
\text { use }\end{array}$} & Adjuvant & 34 & 7 & \multirow{2}{*}{$0.42^{\mathrm{a}}$} \\
\hline & Metastatic & 15 & 1 & \\
\hline \multicolumn{2}{|c|}{ Baseline LVEF (\%)* } & $70.3 \pm 4.3$ & $67.0 \pm 3.1$ & $0.02^{\mathrm{b}}$ \\
\hline \multicolumn{2}{|l|}{ Baseline E/e'* } & $10.8 \pm 2.1$ & $12.1 \pm 3.4$ & $0.50^{\mathrm{b}}$ \\
\hline
\end{tabular}

\section{"Average}

'Fisher's exact probability test, bMann-Whitney U-test

BMI: body mass index, ER: estrogen receptor

E/e': the ratio of early diastolic transmitral flow velocity to mitral annular velocity at the lateral wall 
Table 1B: Comparison of the factors between non-cardiotoxicity group and cardiotoxicity group in non-elderly patients.

\begin{tabular}{|c|c|c|c|c|}
\hline Factors & & $\begin{array}{c}\text { Non- } \\
\text { cardiotoxicity } \\
\text { group/(n=184) }\end{array}$ & $\begin{array}{l}\text { Cardiotoxicity } \\
\text { group/(n=19) }\end{array}$ & $\begin{array}{c}p \\
\text { value }\end{array}$ \\
\hline Age (year) ${ }^{*}$ & & $53.0 \pm 8.3$ & $53.0 \pm 9.0$ & $0.80^{b}$ \\
\hline BMI $\left(\mathrm{kg} / \mathrm{m}^{2}\right)^{\star}$ & & $21.9 \pm 3.8$ & $20.1 \pm 2.1$ & $0.04^{b}$ \\
\hline \multirow{2}{*}{ ER status } & Negative & 102 & 7 & \multirow{2}{*}{$0.12^{\mathrm{a}}$} \\
\hline & Positive & 82 & 12 & \\
\hline \multirow{3}{*}{$\begin{array}{l}\text { Radiation } \\
\text { therapy }\end{array}$} & No & 55 & 8 & \multirow{2}{*}{$0.50^{a}$} \\
\hline & Left & 61 & 6 & \\
\hline & Right & 68 & 5 & \\
\hline \multirow{2}{*}{ Hypertension } & No & 160 & 18 & \multirow{2}{*}{$0.48^{a}$} \\
\hline & Yes & 24 & 1 & \\
\hline \multirow{2}{*}{ Diabetes } & No & 180 & 19 & \multirow{2}{*}{$1.00^{\mathrm{a}}$} \\
\hline & Yes & 4 & 0 & \\
\hline \multirow{2}{*}{ Hyperlipidemia } & No & 150 & 18 & \multirow{2}{*}{$0.21^{a}$} \\
\hline & Yes & 34 & 1 & \\
\hline \multirow{2}{*}{ Hyperuricemia } & No & 178 & 18 & \multirow{2}{*}{$0.50^{\mathrm{a}}$} \\
\hline & Yes & 6 & 1 & \\
\hline \multirow{2}{*}{$\begin{array}{l}\text { Cardiovascular } \\
\text { disease }\end{array}$} & No & 179 & 18 & \multirow{2}{*}{$0.45^{\mathrm{a}}$} \\
\hline & Yes & 5 & 1 & \\
\hline \multirow{2}{*}{$\begin{array}{l}\text { Anthracyclines } \\
\text { use }\end{array}$} & No & 45 & 2 & \multirow{2}{*}{$0.25^{\mathrm{a}}$} \\
\hline & Yes & 139 & 17 & \\
\hline \multirow{2}{*}{ Taxanes use } & No & 54 & 6 & \multirow{2}{*}{$0.84^{a}$} \\
\hline & Yes & 130 & 13 & \\
\hline \multirow{2}{*}{$\begin{array}{l}\text { Trastsuzumab } \\
\text { use }\end{array}$} & Adjuvant & 151 & 17 & \multirow{2}{*}{$0.54^{a}$} \\
\hline & Metastatic & 33 & 2 & \\
\hline $\begin{array}{l}\text { Baseline LVEF } \\
(\%)^{*}\end{array}$ & & $68.0 \pm 4.8$ & $63.7 \pm 3.9$ & $<0.01 b$ \\
\hline Baseline E/e'* & & $9.0 \pm 2.2$ & $7.9 \pm 1.8$ & $0.05 b$ \\
\hline
\end{tabular}

${ }^{*}$ Average

aFisher's exact probability test

'Mann-Whitney U-test a significant associated factor. The predictive accuracy of the baseline LVEF values for trastuzumab-related cardiotoxicity was then determined by measuring the sensitivity, specificity, and area under the Receiver Operating Characteristic (ROC) curve (Figure 1). Based on the ROC curve, the optimal cutoff value of baseline LVEF as an indicator for trastuzumab-related cardiotoxicity in elderly patients was projected to be $67 \%$, which yielded a sensitivity of $62 \%$ and a specificity of $82 \%$, with the area under the curve a 0.77 (Figure 1A). On the other hand, the cutoff value of baseline LVEF in non-elderly patients was $66 \%$, which had a sensitivity of $79 \%$ and a specificity of $66 \%$, with the area under the curve at 0.76 (Figure 1B).

Table 3 shows the result of multivariate analysis including baseline LVEF and other established relatively associated factors. In elderly patients, there was an increased prediction ability of cardiotoxicity associated with a lower baseline LVEF value $<67 \%$ (OR 5.59, 95\% CI: 1.08-33.24; $p=0.04$ ) after adjusting for the above possible confounders. Similarly, in nonelderly patients, a lower baseline LVEF value $<66 \%$ (OR 4.61, 95\% CI: $1.48-17.50 ; p<0.01)$ was significantly associated with trastuzumab-related cardiotoxicity.

\section{Discussion}

Understanding the risk factors for cardiotoxicity is very important for safer treatment with trastuzumab in breast cancer patients. In the current study, we demonstrated that the baseline LVEF level was the most significant risk factor for trastuzumab-related cardiotoxicity. Interestingly, each predictive value for cardiotoxicity of baseline LVEF in elderly and non-elderly patients was found to be approximately similar. To the best of our knowledge, there are no reports prior to this study comparing the risk factors in non-elderly with that in

Table 2: Univariate logistic regression analysis to identify the risk factors for cardiotoxicity induced by trastuzumab in elderly and non-elderly patients.

\begin{tabular}{|c|c|c|c|c|c|c|c|}
\hline \multirow{2}{*}{ Factors } & & \multicolumn{3}{|c|}{ Elderly patients } & \multicolumn{3}{|c|}{ Non-elderly patients } \\
\hline & & Odds ratio & $95 \% \mathrm{CI}$ & $p$ value & Odds ratio & $95 \% \mathrm{Cl}$ & $p$ value \\
\hline Age (year) & Increase & 0.98 & $0.81-1.14$ & 0.8 & 1.01 & $0.95-1.07$ & 0.8 \\
\hline BMI $\left(\mathrm{kg} / \mathrm{m}^{2}\right)$ & Increase & 0.99 & $0.80-1.22$ & 0.95 & 0.83 & $0.69-0.97$ & 0.02 \\
\hline ER status & ER+/ER- & 1.6 & $0.35-8.49$ & 0.54 & 2.13 & $0.82-5.95$ & 0.12 \\
\hline \multirow{3}{*}{ Radiation therapy } & Left/No & 0.34 & $0.02-2.41$ & 0.3 & 0.68 & $0.21-2.06$ & 0.49 \\
\hline & Right/No & 0.63 & $0.08-3.37$ & 0.6 & 0.5 & $0.14-1.60$ & 0.25 \\
\hline & Left/Right & 0.54 & $0.02-6.28$ & 0.62 & 1.33 & $0.38-4.85$ & 0.64 \\
\hline Hypertension & Yes/No & 1.13 & $0.21-5.19$ & 0.88 & 0.37 & $0.02-1.93$ & 0.28 \\
\hline Diabetes & Yes/No & 1.02 & $0.05-7.39$ & 0.98 & $<0.01$ & $0.00-6.11$ & 0.37 \\
\hline Hyperlipidemia & Yes/No & 1.03 & $0.19-4.73$ & 0.97 & 0.24 & $0.01-1.25$ & 0.1 \\
\hline Hyperuricemia & Yes/No & 0.63 & $0.03-4.25$ & 0.67 & 1.65 & $0.08-10.41$ & 0.67 \\
\hline Cardiovascular disease & Yes/No & 3.43 & $0.60-17.63$ & 0.16 & 1.98 & $0.10-13.25$ & 0.57 \\
\hline Anthracyclines use & Yes/No & 2.42 & $0.53-12.88$ & 0.25 & 2.75 & $0.75-17.77$ & 0.14 \\
\hline Taxanes use & Yes/No & 0.63 & $0.13-2.97$ & 0.55 & 0.9 & $0.33-2.67$ & 0.84 \\
\hline Trastuzumab use & Adjuvant/Metastatic & 3.08 & $0.49-60.35$ & 0.26 & 1.85 & $0.50-12.07$ & 0.39 \\
\hline Baseline LVEF (\%) & decrease & 1.18 & $1.00-1.43$ & 0.05 & 1.17 & $1.07-1.30$ & $<0.01$ \\
\hline Baseline E/e' & increase & 0.99 & $0.83-1.07$ & 0.92 & 0.75 & $0.54-0.98$ & 0.03 \\
\hline
\end{tabular}

Citation: Kittaka N, Mukai M, Fukui R, Hashimoto Y, Ishitobi M, et al. (2018) Comparison of Risk Factors for Trastuzumab-Related 
Table 3: Multivariate logistic regression analysis to identify the risk factors for cardiotoxicity induced by trastuzumab in elderly and non-elderly patients.

\begin{tabular}{|c|c|c|c|c|}
\hline \multicolumn{2}{|c|}{ Factors } & Odds ratio & $95 \% \mathrm{Cl}$ & $p$ value \\
\hline \multicolumn{5}{|c|}{ Multivariate analysis in elderly patients } \\
\hline Cardiovascular disease & Yes/No & 2.86 & $0.42-17.85$ & 0.27 \\
\hline Anthracyclines use & Yes/No & 2.21 & $0.40-14.45$ & 0.36 \\
\hline Trastuzumab use & Adjuvant/Metastatic & 2.25 & $0.30-46.30$ & 0.26 \\
\hline Baseline LVEF (\%) & Less than $67 \%$ & 5.59 & $1.08-33.24$ & 0.04 \\
\hline BMI $\left(\mathrm{kg} / \mathrm{m}^{2}\right)$ & Increase & 1 & $0.85-1.16$ & 0.96 \\
\hline \multicolumn{5}{|c|}{ Multivariate analysis in non-elderly patients } \\
\hline BMI $\left(\mathrm{kg} / \mathrm{m}^{2}\right)$ & Increase & 1 & $0.85-1.16$ & 0.96 \\
\hline Baseline LVEF (\%) & less than $66 \%$ & 4.61 & $1.48-17.50$ & $<0.01$ \\
\hline Baseline E/e' & Increase & 0.84 & $0.61-1.08$ & 0.19 \\
\hline
\end{tabular}
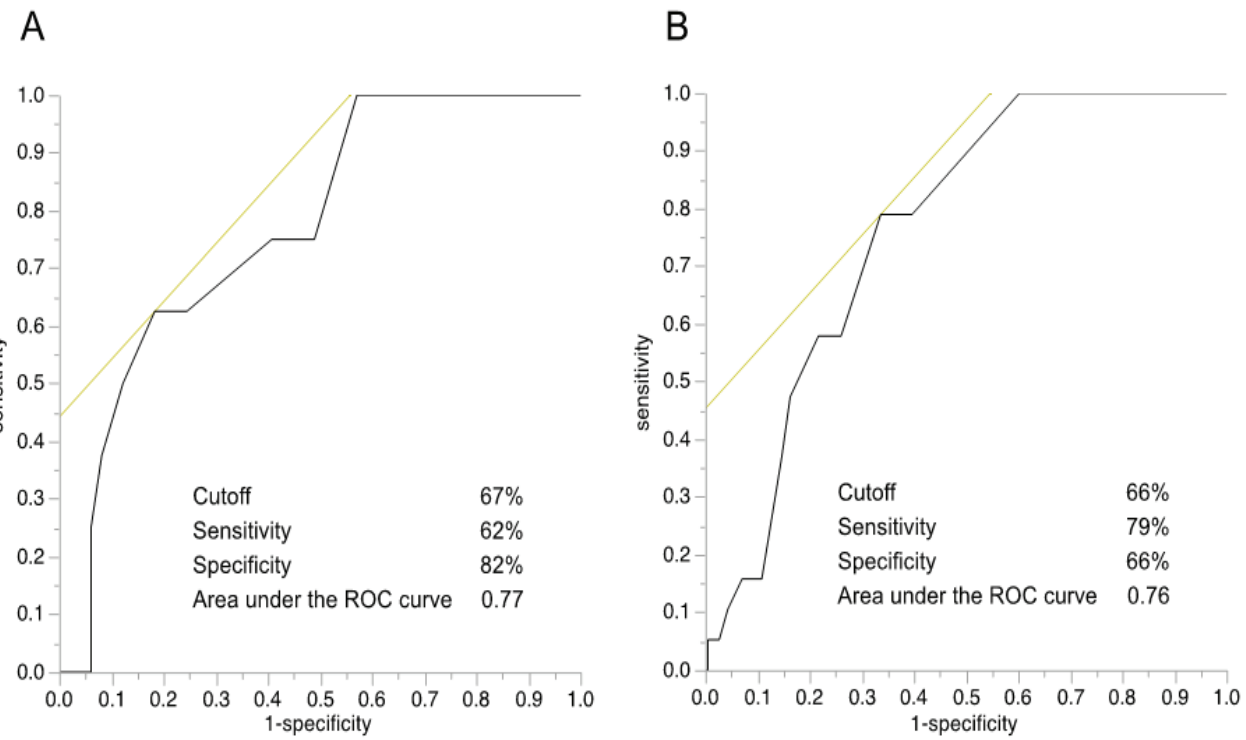

Figure 1: Receiver Operating Characteristic (ROC) curve for baseline LVEF values to predict trastuzumab-related cardiotoxicity in elderly (A) and non-elderly (B) patients. (A) ROC analysis showed that the optimal cutoff value for change in baseline LVEF value was $67 \%$. (B) ROC analysis showed that the optimal cutoff value for change in baseline LVEF value was $66 \%$.

the elderly. This is the first report thus far demonstrating that a low value of baseline LVEF is the common risk factor for trastuzumab-related cardiotoxicity in elderly and non-elderly breast cancer patients.

Previously, Bowels et al. showed that the risk of cardiotoxicity was associated with adjuvant anthracycline and/or trastuzumab in a population-based cohort study [6]. Chavez-MacGregor et al reported that older age, coronary artery disease, hypertension, and weekly trastuzumab administration increased the risk of cardiotoxicity among trastuzumab-treated patients [7]. In the studies investigating risk factors in elderly patients, history of cardiac disease, diabetes, hypertension, obesity, and prior anthracycline use were associated with a higher incidence of cardiotoxicity $[8,9]$. In our study, history of cardiac disease and prior anthracycline use were also identified as risk factors that were likely to be associated with cardiotoxicity in elderly patients. These findings are similar to those seen in previous reports $[8,9]$. However, in multivariate analysis, we found that a lower baseline LVEF value was the most significant factor in both elderly and non-elderly patients. This result was different from those reports, because patients with low values of baseline LVEF may be excluded in such clinical trials. The baseline LVEF may be conveniently evaluated by means of echocardiography, and is considered a superior predictive marker of cardiotoxicity [17] than others. It may thus be useful to assess whether the treatment of trastuzumab-based chemotherapy is safely performed for the patient. Patients with low LVEF at baseline have to undergo careful monitoring of cardiac activity for a certain period. Chaves-MacGregor et al., recommends increasing the rates of cardiac monitoring for elderly patients, and adequate cardiac monitoring among trastuzumab-treated patients should be considered a marker of quality of care [18]. However, no clear universal guidelines for cardiac monitoring exist for patients receiving trastuzumabbased chemotherapy. For instance, the Canadian Trastuzumab Working Group recommends that carefully selected patients receive a clinical and LVEF assessment at baseline and every three months during treatment [19]. Whereas, Hart A et al., suggest that routine intensive monitoring for cardiac function is not needed after completion of trastuzumab treatment [20]. 
There are several limitations to this study. One limitation is that serum biomarkers such as Troponin I and N-terminal pro- $\mathrm{B}$ type natriuretic peptide (NT pro-BNP) were not included in the examination risk factors. Troponin I is a well-established early serum biomarker of myocardial necrosis with high diagnostic and high prognostic values $[13,21]$. NT pro-BNP levels may be used to identify the risk of developing cardiac dysfunction during trastuzumab-based treatment [22]. As such, it will be necessary to examine more kinds of risk factors including serum biomarkers in future. Another limitation is that the possibility of selection bias as some elderly patients with low LVEF at baseline have not been treated with trastuzumab-based chemotherapy, but we believe that this result might reflect real data in the routine clinical care for breast cancer.

In this study, we showed that a lower value of baseline LVEF is the common risk factor of trastuzumab-related cardiotoxicity both in elderly and non-elderly breast cancer patients. We conclude that if cardiac function is maintained, even elderly might be able patients to receive trastuzumabbased chemotherapy under careful observation.

\section{Conflicts of interest}

We declare no potential conflicts of interest.

\section{References}

1. Yamauchi H, Stearns V, Hayes DF (2001) When is a tumor marker ready for prime time? A case study of c-erbB-2 as a predictive factor in breast cancer. J Clin Oncol 19: 2334-2356.

2. Slamon D, Eiermann W, Robert N, Pienkowski T, Martin M, et al. (2011) Adjuvant trastuzumab in HER2-positive breast cancer. N Engl J Me 365: 1273-1283.

3. Slamon DJ, Jones- LB, Shak S, Fuchs H, Paton V, et al. (2001) Use of chemotherapy plus a monoclonal antibody against HER2 for metastatic breast cancer that overexpresses HER2. N Engl J Med 344: 783-792.

4. Yeh ET, Bickford CL (2009) Cardiovascular complications of cancer therapy: incidence, pathogenesis, diagnosis, and management. J Am Coll Cardiol 53: 2231-2347.

5. Romond EH, Perez EA, Bryant J, Suman VJ, Geyer CE Jr, et al. (2005) Trastuzumab plus adjuvant chemotherapy for operable HER2-positive breast cancer. N Engl J Med 353: 1673-1684.

6. Bowles EJ, Wellman R, Feigelson HS, Onitilo AA, Freedman AN, et al. (2012) Risk of heart failure in breast cancer patients after anthracycline and trastuzumab treatment: a retrospective cohort study. J Natl Cancer Inst 104: 1293-1305.

7. MacGregor- CM, Zhang N, Buchholz TA, Zhang $\mathrm{Y}$, Niu J, et al. (2013) Trastuzumab-Related Cardiotoxicity Among Older Patients With Breast Cancer. J Clin Oncol 31: 4222-4228.

8. Serrano C, Cortes J, Arruda-DM L, Bellet M, Gomez P, et al. (2012) Trastuzumab-related cardiotoxicity in the elderly: a role for cardiovascular risk factors. Ann Oncol 23: 897-902.

9. Adamo V, Ricciardi GR, Adamo B, Ferraro G, Franchina T, et al. (2014) The risk of toxicities from trastuzumab, alone or in combination, in an elderly breast cancer population. Oncology 86: 16-21.
10. Nishimura RA, Otto CM, Bonow RO, Carabello BA, Erwin JP 3rd, et al. (2014) 2014 AHA/ACC Guideline for the Management of Patients With Valvular Heart Disease: executive summary: a report of the American College of Cardiology/American Heart Association Task Force on Practice Guidelines. Circulation 129: 2440-2492.

11. Priori SG, Lundqvist- BC, Mazzanti A, Blom N, Borggrefe M, et al. (2015) 2015 ESC Guidelines for the management of patients with ventricular arrhythmias and the prevention of sudden cardiac death: The Task Force for the Management of Patients with Ventricular Arrhythmias and the Prevention of Sudden Cardiac Death of the European Society of Cardiology (ESC). Endorsed by: Association for European Paediatric and Congenital Cardiology (AEPC). Eur Heart J 36: 2793-2867.

12. Suter TM, Procter $M$, Veldhuisen-VDJ, Muscholl $M$, Bergh J, et al. (2007) Trastuzumab-associated cardiac adverse effects in the herceptin adjuvant trial. J Clin Oncol 25: 3859-3865.

13. Cardinale D, Colombo A, Torrisi R, Sandri MT, Civelli M, et al. Trastuzumab-induced cardiotoxicity: clinical and prognostic implications of troponin I evaluation. J Clin Oncol 28: 3910-3916.

14. Aapro M, Johnson CD (2007) The elderly: geriatric oncology finally deserving adequate attention. Eur J Cancer 43: 2312-2314.

15. Plana JC, Galderisi M, Barac A, Ewer MS, Ky B, et al. Expert consensus for multimodality imaging evaluation of adult patients during and after cancer therapy: a report from the American Society of Echocardiography and the European Association of Cardiovascular Imaging. J Am Soc Echocardiogr 27: 911-939.

16. Dores H, Abecasis J, Correia MJ, Gandara F, Fonseca C, et al. (2013) Detection of early sub-clinical trastuzumab-induced cardiotoxicity in breast cancer patients. Arq Bras Cardiol 100: 328-332.

17. Chiu-TE, Yothers $G$, Romond E, Geyer CE Jr, Ewer M, et al. (2005) Assessment of cardiac dysfunction in a randomized trial comparing doxorubicin and cyclophosphamide followed by paclitaxel, with or without trastuzumab as adjuvant therapy in node-positive, human epidermal growth factor receptor 2-overexpressing breast cancer: NSABP B-31. J Clin Oncol 23: 7811-7819.

18. MacGregor-CM, Niu J, Zhang N, Elting LS, Smith BD, et al. (2015) Cardiac Monitoring During Adjuvant Trastuzumab-Based Chemotherapy Among Older Patients With Breast Cancer. J Clin Oncol 33: 2176-2183.

19. Mackey JR, Clemons M, Cote MA, Delgado D, Dent S, et al. (2008) Cardiac management during adjuvant trastuzumab therapy: recommendations of the Canadian Trastuzumab Working Group. Curr Oncol 15: 24-35.

20. Goldhar HA, Yan AT, Ko DT, Earle CC, Tomlinson GA, et al. (2016) The Temporal Risk of Heart Failure Associated With Adjuvant Trastuzumab in Breast Cancer Patients: A Population Study. J Natl Cancer Inst 108.

21. Cardinale D, Sandri MT, Colombo A, Colombo N, Boeri M, et al. (2004) Prognostic value of troponin I in cardiac risk stratification of cancer patients undergoing high-dose chemotherapy. Circulation 109: 2749-2754.

22. Goel S, Simes RJ, Beith JM (2011) Exploratory analysis of cardiac biomarkers in women with normal cardiac function receiving trastuzumab for breast cancer. Asia Pac J Clin Oncol 7: 276-280. 